Acta Crystallographica Section E

Structure Reports

Online

ISSN 1600-5368

Editors: W. Clegg and D. G. Watson

\title{
(6-Acetoxy-2-acetylphenyl- $\left.\kappa^{2} C^{1}, O^{1}\right)$ tetracarbonylmanganese(I)
}

\author{
Victor D. Fester, Lyndsay Main and Brian K. Nicholson
}

Copyright $($ International Union of Crystallography

Author(s) of this paper may load this reprint on their own web site or institutional repository provided that this cover page is retained. Republication of this article or its storage in electronic databases other than as specified above is not permitted without prior permission in writing from the IUCr.

For further information see http://journals.iucr.org/services/authorrights.html 


\section{metal-organic compounds}

Acta Crystallographica Section E

\section{Structure Reports}

Online

ISSN $1600-5368$

\section{(6-Acetoxy-2-acetylphenyl- $\left.\kappa^{2} C^{1}, O^{1}\right)$ - tetracarbonylmanganese(I)}

\section{Victor D. Fester, Lyndsay Main and Brian K. Nicholson*}

Chemistry Department, University of Waikato, Private Bag 3105, Hamilton 3240 New Zealand

Correspondence e-mail: b.nicholson@waikato.ac.nz

Received 18 September 2007; accepted 27 September 2007

Key indicators: single-crystal X-ray study; $T=168 \mathrm{~K}$; mean $\sigma(\mathrm{C}-\mathrm{C})=0.003 \AA$; $R$ factor $=0.026 ; w R$ factor $=0.073 ;$ data-to-parameter ratio $=12.7$.

The title compound, $\left[\mathrm{Mn}\left(\mathrm{C}_{10} \mathrm{H}_{9} \mathrm{O}_{3}\right)(\mathrm{CO})_{4}\right]$, is formed by orthomanganation of $3^{\prime}$-acetoxyacetophenone at the sterically crowded ortho site. The atoms of the benzene and the cyclometallated rings are coplanar to within $<0.018 \AA$, and there are no significant intramolecular interactions between the $\mathrm{Mn}(\mathrm{CO})_{4}$ group and the adjacent acetoxy group.

\section{Related literature}

The preparation and structures of related orthomanganated aryl ketones have been reviewed (Main \& Nicholson, 1994). Preference in cyclometallation reactions for the more sterically crowded isomer for other $3^{\prime}$-substituted acetophenones has been observed (Cooney et al., 1988, 2001; Liebeskind et al., 1989).

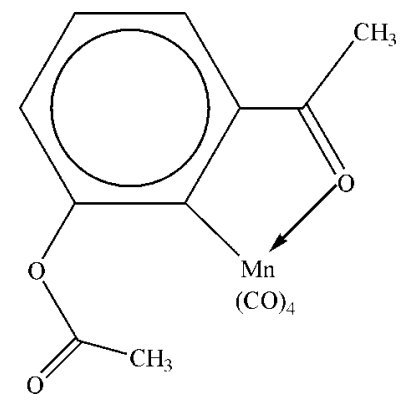

\section{Experimental}

Crystal data

$\left[\mathrm{Mn}\left(\mathrm{C}_{10} \mathrm{H}_{9} \mathrm{O}_{3}\right)(\mathrm{CO})_{4}\right]$
$M_{r}=344.15$
Triclinic, $P \overline{1}$
$a=9.306(2) \AA$
$b=9.635(2) \AA$
$c=9.954(2) \AA$
$\alpha=68.594(3)^{\circ}$
$\beta=67.663(3)^{\circ}$

$\gamma=68.422(3)^{\circ}$ $V=740.9(3) \AA^{3}$ $Z=2$

Mo $K \alpha$ radiation $\mu=0.92 \mathrm{~mm}^{-1}$

$T=168$ (2) K

$0.72 \times 0.65 \times 0.15 \mathrm{~mm}$

Data collection

Bruker SMART CCD diffractometer

Absorption correction: multi-scan (SADABS; Sheldrick, 1998)

$T_{\min }=0.711, T_{\max }=0.870$

9684 measured reflections 2988 independent reflections 2730 reflections with $I>2 \sigma(I)$ $R_{\text {int }}=0.015$

Refinement

$R\left[F^{2}>2 \sigma\left(F^{2}\right)\right]=0.026$

$w R\left(F^{2}\right)=0.073$

$S=1.02$

2988 reflections

235 parameters

All $\mathrm{H}$-atom parameters refined

$\Delta \rho_{\max }=0.26{\mathrm{e} \AA^{-3}}^{-3}$

$\Delta \rho_{\min }=-0.41{\mathrm{e} \AA^{-3}}^{-3}$

Data collection: SMART (Bruker, 2001); cell refinement: SAINT (Bruker, 2001); data reduction: $S A I N T$; $\operatorname{program}(\mathrm{s})$ used to solve structure: SHELXS97 (Sheldrick, 1997); program(s) used to refine structure: SHELXL97 (Sheldrick, 1997); molecular graphics: ORTEP-3 for Windows (Farrugia, 1997); software used to prepare material for publication: WinGX (Farrugia, 1999).

The authors thank Dr Jan Wikaira, University of Canterbury, for collection of X-ray intensity data.

Supplementary data and figures for this paper are available from the IUCr electronic archives (Reference: FJ2041).

\section{References}

Bruker (2001). SMART and SAINT. Bruker AXS Inc., Madison, Wisconsin, USA.

Cooney, J. M., Gommans, L. H. P., Main, L. \& Nicholson, B. K. (1988). J. Organomet. Chem. 349, 197-208.

Cooney, J. M., Gommans, L. H. P., Main, L. \& Nicholson, B. K. (2001). J. Organomet. Chem. 634, 157-166.

Farrugia, L. J. (1997). J. Appl. Cryst. 30, 565.

Farrugia, L. J. (1999). J. Appl. Cryst. 32, 837-838.

Liebeskind, L. S., Gasdaska, J. R., McCallum, J. S. \& Tremont, S. J. (1989). J. Org. Chem. 54, 669-677.

Main, L. \& Nicholson, B. K. (1994). Adv. Met.-Org. Chem. 3, 1-50.

Sheldrick, G. M. (1997). SHELXS97 and SHELXL97. University of Göttingen, Germany.

Sheldrick, G. M. (1998). SADABS. University of Göttingen, Germany. 


\section{supplementary materials}




\section{supplementary materials}

Acta Cryst. (2007). E63, m2655 [ doi:10.1107/S1600536807047605 ]

(6-Acetoxy-2-acetylphenyl- $\left.\kappa^{2} C^{1}, o^{1}\right)$ tetracarbonylmanganese(I)

\section{D. Fester, L. Main and B. K. Nicholson}

\section{Comment}

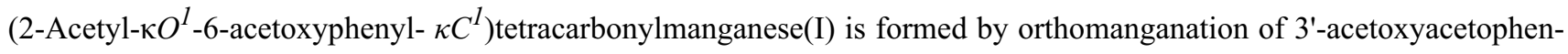
one at the sterically crowded ortho site. The atoms of the phenyl and the cyclometallated rings are coplanar to within $<0.018$ $\AA$, and there are no significant intramolecular interactions between the $\mathrm{Mn}(\mathrm{CO})_{4}$ group and the adjacent acetoxy one. The Mn1 - C12 distance trans to O1 (1.8046 (18) $\AA$ ) is shorter than the Mn1-C11 distance trans to C1 (1.8367 (19) $\AA$ ), while the two $\mathrm{Mn}-\mathrm{CO}$ distances trans to each other are the longest (av. 1.857 (2) $\AA$ ). The $\mathrm{Mn} 1-\mathrm{C} 1$ distance is significantly shorter than the Mn1—O1 distance (2.0445 (15) and 2.0548 (11) $\AA$ respectively) contrary to the trend expected purely on covalent radii. These conform to the pattern found for other orthomanganated arenes (Main and Nicholson, 1994).

\section{Experimental}

The title compound was prepared by reaction of $\mathrm{PhCH}_{2} \mathrm{Mn}(\mathrm{CO})_{5}$ with 3'-acetoxyacetophenone in refluxing heptane, under the usual conditions (Main and Nicholson, 1994). The structure was determined to confirm that cyclometallation had occurred at the sterically crowded 2'-position, rather than the alternative 6'-position, and to detect any residual interactions with the adjacent acetoxy group which might have directed this preference.

\section{Refinement}

All H-atoms were located as the highest peaks in a penultimate difference map and were refined with isotropic temperature factors.

\section{Figures}

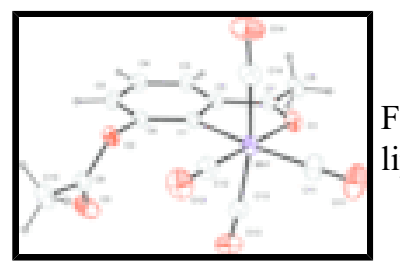

Fig. 1. Structure of (2-Acetyl- $\kappa O^{l}$-6-acetoxyphenyl- $\kappa C^{l}$ )tetracarbonylmanganese(I), with ellipsoids drawn at the $30 \%$ probability level.

(6-Acetoxy-2-acetylphenyl- $\left.{ }^{2} C^{1}, O^{1}\right)$ tetracarbonylmanganese(I)

\section{Crystal data}

$\left[\mathrm{Mn}\left(\mathrm{C}_{10} \mathrm{H}_{9} \mathrm{O}_{3}\right)(\mathrm{CO})_{4}\right]$

$M_{r}=344.15$

$Z=2$

Triclinic, $P \mathrm{~T}$

$F_{000}=348$

$D_{\mathrm{x}}=1.543 \mathrm{Mg} \mathrm{m}^{-3}$ 


\section{supplementary materials}

Hall symbol: -P 1

$$
\begin{aligned}
& a=9.306(2) \AA \\
& b=9.635(2) \AA \\
& c=9.954(2) \AA \\
& \alpha=68.594(3)^{\mathrm{o}} \\
& \beta=67.663(3)^{\mathrm{o}} \\
& \gamma=68.422(3)^{\mathrm{o}} \\
& V=740.9(3) \AA^{3}
\end{aligned}
$$

\section{Data collection}

Bruker SMART CCD diffractometer

Radiation source: fine-focus sealed tube Monochromator: graphite

$T=168(2) \mathrm{K}$

$\omega$ scans

Absorption correction: multi-scan

(SADABS; Sheldrick, 1998)

$T_{\min }=0.711, T_{\max }=0.870$

9684 measured reflections

\section{Refinement}

Refinement on $F^{2}$

Least-squares matrix: full

$R\left[F^{2}>2 \sigma\left(F^{2}\right)\right]=0.026$

$w R\left(F^{2}\right)=0.073$

$S=1.02$

2988 reflections

235 parameters

Primary atom site location: structure-invariant direct methods
Mo $K \alpha$ radiation

$\lambda=0.71073 \AA$

Cell parameters from 3920 reflections

$\theta=2-26^{\circ}$

$\mu=0.92 \mathrm{~mm}^{-1}$

$T=168$ (2) K

Block, yellow

$0.72 \times 0.65 \times 0.15 \mathrm{~mm}$

$$
\begin{aligned}
& 2730 \text { reflections with } I>2 \sigma(I) \\
& R_{\text {int }}=0.015 \\
& \theta_{\max }=26.4^{\circ} \\
& \theta_{\min }=2.3^{\circ} \\
& h=-11 \rightarrow 11 \\
& k=-12 \rightarrow 11 \\
& l=-12 \rightarrow 12
\end{aligned}
$$

Secondary atom site location: difference Fourier map Hydrogen site location: difference Fourier map All H-atom parameters refined

$$
\begin{aligned}
& w=1 /\left[\sigma^{2}\left(F_{\mathrm{o}}^{2}\right)+(0.0452 P)^{2}+0.1914 P\right] \\
& \text { where } P=\left(F_{\mathrm{o}}{ }^{2}+2 F_{\mathrm{c}}{ }^{2}\right) / 3 \\
& (\Delta / \sigma)_{\max }=0.004 \\
& \Delta \rho_{\max }=0.26 \mathrm{e} \AA^{-3} \\
& \Delta \rho_{\min }=-0.41 \mathrm{e} \AA^{-3}
\end{aligned}
$$

Extinction correction: none

Fractional atomic coordinates and isotropic or equivalent isotropic displacement parameters $\left(\hat{A}^{2}\right)$

$\begin{array}{lllll} & x & y & z & U_{\text {iso }} * / U_{\text {eq }} \\ \text { Mn1 } & 0.15624(3) & 0.30950(3) & 0.24687(2) & 0.03251(9) \\ \text { C1 } & 0.37221(17) & 0.14695(17) & 0.24738(15) & 0.0287(3) \\ \text { C2 } & 0.46885(18) & 0.18432(17) & 0.30330(16) & 0.0311(3) \\ \text { C3 } & 0.6217(2) & 0.09150(19) & 0.31439(19) & 0.0380(3) \\ \text { C4 } & 0.6832(2) & -0.0424(2) & 0.2682(2) & 0.0428(4) \\ \text { C5 } & 0.5919(2) & -0.08368(19) & 0.21356(19) & 0.0389(3) \\ \text { C6 } & 0.44005(18) & 0.00979(17) & 0.20521(16) & 0.0314(3) \\ \text { C7 } & 0.3934(2) & 0.32656(18) & 0.35101(16) & 0.0337(3) \\ \text { C8 } & 0.4716(3) & 0.3870(2) & 0.4155(2) & 0.0425(4)\end{array}$




\section{supplementary materials}

$\begin{array}{lllll}\text { C9 } & 0.29739(19) & -0.15989(18) & 0.22594(19) & 0.0378(3) \\ \text { C10 } & 0.2255(3) & -0.2011(2) & 0.1395(3) & 0.0512(5) \\ \text { C11 } & -0.0262(2) & 0.4716(2) & 0.2641(2) & 0.0509(4) \\ \text { C12 } & 0.0719(2) & 0.2290(2) & 0.1657(2) & 0.0415(4) \\ \text { C13 } & 0.08616(19) & 0.1809(2) & 0.43689(19) & 0.0395(4) \\ \text { C14 } & 0.2643(2) & 0.4043(2) & 0.0558(2) & 0.0470(4) \\ \text { O1 } & 0.25602(14) & 0.39925(12) & 0.33756(13) & 0.0383(3) \\ \text { O2 } & 0.35525(14) & -0.03417(12) & 0.14249(12) & 0.0359(2) \\ \text { O3 } & 0.30441(17) & -0.22465(16) & 0.35196(16) & 0.0559(4) \\ \text { O11 } & -0.1391(2) & 0.5708(2) & 0.2727(2) & 0.0809(5) \\ \text { O12 } & 0.01425(17) & 0.18291(17) & 0.11287(17) & 0.0599(4) \\ \text { O13 } & 0.05411(16) & 0.09520(18) & 0.55063(15) & 0.0572(4) \\ \text { O14 } & 0.3357(2) & 0.4562(2) & -0.06173(17) & 0.0813(5) \\ \text { H3 } & 0.679(2) & 0.117(2) & 0.353(2) & 0.046(5)^{*} \\ \text { H4 } & 0.788(2) & -0.112(2) & 0.276(2) & 0.048(5)^{*} \\ \text { H5 } & 0.629(2) & -0.178(2) & 0.182(2) & 0.044(5)^{*} \\ \text { H81 } & 0.574(3) & 0.399(3) & 0.353(3) & 0.075(8)^{*} \\ \text { H82 } & 0.489(3) & 0.321(3) & 0.506(3) & 0.063(6)^{*} \\ \text { H83 } & 0.406(3) & 0.481(3) & 0.435(2) & 0.059(6)^{*} \\ \text { H101 } & 0.304(3) & -0.215(3) & 0.049(3) & 0.081(8)^{*} \\ \text { H102 } & 0.143(3) & -0.120(3) & 0.113(3) & 0.072(7)^{*} \\ \text { H103 } & 0.185(3) & -0.288(4) & 0.196(3) & 0.093(9)^{*} \\ & & & & \end{array}$

Atomic displacement parameters $\left(\AA^{2}\right)$

$\begin{array}{lllllll} & U^{11} & U^{22} & U^{33} & U^{12} & U^{13} & U^{23} \\ \mathrm{Mn} 1 & 0.03310(14) & 0.02938(14) & 0.03785(15) & -0.00427(9) & -0.01615(10) & -0.00960(10) \\ \text { C1 } & 0.0313(7) & 0.0291(7) & 0.0266(7) & -0.0100(6) & -0.0102(6) & -0.0042(5) \\ \text { C2 } & 0.0357(7) & 0.0310(7) & 0.0297(7) & -0.0133(6) & -0.0121(6) & -0.0039(6) \\ \text { C3 } & 0.0358(8) & 0.0409(9) & 0.0417(8) & -0.0121(7) & -0.0175(7) & -0.0069(7) \\ \text { C4 } & 0.0341(8) & 0.0444(9) & 0.0480(9) & -0.0038(7) & -0.0168(7) & -0.0115(8) \\ \text { C5 } & 0.0402(8) & 0.0337(8) & 0.0406(8) & -0.0032(7) & -0.0123(7) & -0.0129(7) \\ \text { C6 } & 0.0374(8) & 0.0307(7) & 0.0289(7) & -0.0109(6) & -0.0115(6) & -0.0068(6) \\ \text { C7 } & 0.0448(8) & 0.0304(7) & 0.0302(7) & -0.0155(6) & -0.0148(6) & -0.0030(6) \\ \text { C8 } & 0.0582(11) & 0.0383(9) & 0.0444(10) & -0.0193(8) & -0.0241(9) & -0.0089(8) \\ \text { C9 } & 0.0356(8) & 0.0287(7) & 0.0500(10) & -0.0049(6) & -0.0148(7) & -0.0124(7) \\ \text { C10 } & 0.0623(12) & 0.0427(10) & 0.0631(13) & -0.0209(10) & -0.0232(11) & -0.0170(9) \\ \text { C11 } & 0.0477(10) & 0.0448(10) & 0.0624(12) & 0.0007(8) & -0.0264(9) & -0.0182(9) \\ \text { C12 } & 0.0398(8) & 0.0392(9) & 0.0482(9) & -0.0061(7) & -0.0203(7) & -0.0105(7) \\ \text { C13 } & 0.0323(8) & 0.0479(10) & 0.0430(9) & -0.0094(7) & -0.0142(7) & -0.0147(8) \\ \text { C14 } & 0.0565(10) & 0.0435(10) & 0.0458(10) & -0.0138(8) & -0.0259(9) & -0.0045(8) \\ \text { O1 } & 0.0462(6) & 0.0299(5) & 0.0451(6) & -0.0062(5) & -0.0207(5) & -0.0128(5) \\ \text { O2 } & 0.0471(6) & 0.0323(6) & 0.0362(6) & -0.0136(5) & -0.0170(5) & -0.0091(4) \\ \text { O3 } & 0.0630(8) & 0.0504(8) & 0.0587(8) & -0.0268(7) & -0.0330(7) & 0.0092(6) \\ \text { O11 } & 0.0606(9) & 0.0622(10) & 0.1086(14) & 0.0228(8) & -0.0392(9) & -0.0332(10) \\ \text { O12 } & 0.0597(8) & 0.0649(9) & 0.0777(10) & -0.0146(7) & -0.0405(8) & -0.0235(8) \\ \text { O13 } & 0.0491(7) & 0.0742(10) & 0.0447(7) & -0.0256(7) & -0.0133(6) & -0.0023(7) \\ \text { O14 } & 0.0975(13) & 0.0914(13) & 0.0457(9) & -0.0431(11) & -0.0189(8) & 0.0090(8)\end{array}$




\section{supplementary materials}

Geometric parameters $\left(\AA,{ }^{\circ}\right)$

\begin{tabular}{|c|c|c|c|}
\hline $\mathrm{Mn} 1-\mathrm{C} 12$ & $1.8046(18)$ & $\mathrm{C} 6-\mathrm{O} 2$ & $1.4161(18)$ \\
\hline $\mathrm{Mn} 1-\mathrm{C} 11$ & $1.8367(19)$ & $\mathrm{C} 7-\mathrm{O} 1$ & $1.243(2)$ \\
\hline $\mathrm{Mn} 1-\mathrm{C} 14$ & $1.849(2)$ & $\mathrm{C} 7-\mathrm{C} 8$ & $1.490(2)$ \\
\hline $\mathrm{Mn} 1-\mathrm{C} 13$ & $1.8665(18)$ & $\mathrm{C} 8-\mathrm{H} 83$ & $0.93(2)$ \\
\hline $\mathrm{Mn} 1-\mathrm{C} 1$ & $2.0443(15)$ & $\mathrm{C} 8-\mathrm{H} 82$ & $0.93(2)$ \\
\hline $\mathrm{Mn} 1-\mathrm{O} 1$ & $2.0548(11)$ & $\mathrm{C} 8-\mathrm{H} 81$ & $0.94(3)$ \\
\hline $\mathrm{C} 1-\mathrm{C} 6$ & $1.382(2)$ & $\mathrm{C} 9-\mathrm{O} 3$ & $1.195(2)$ \\
\hline $\mathrm{C} 1-\mathrm{C} 2$ & $1.419(2)$ & $\mathrm{C} 9-\mathrm{O} 2$ & $1.3611(19)$ \\
\hline $\mathrm{C} 2-\mathrm{C} 3$ & $1.395(2)$ & $\mathrm{C} 9-\mathrm{C} 10$ & $1.493(2)$ \\
\hline $\mathrm{C} 2-\mathrm{C} 7$ & $1.453(2)$ & $\mathrm{C} 10-\mathrm{H} 101$ & $0.94(3)$ \\
\hline $\mathrm{C} 3-\mathrm{C} 4$ & $1.376(2)$ & $\mathrm{C} 10-\mathrm{H} 102$ & $0.91(3)$ \\
\hline $\mathrm{C} 3-\mathrm{H} 3$ & $0.90(2)$ & $\mathrm{C} 10-\mathrm{H} 103$ & $0.94(3)$ \\
\hline $\mathrm{C} 4-\mathrm{C} 5$ & $1.391(2)$ & $\mathrm{C} 11-\mathrm{O} 11$ & $1.131(2)$ \\
\hline $\mathrm{C} 4-\mathrm{H} 4$ & $0.97(2)$ & $\mathrm{C} 12-\mathrm{O} 12$ & $1.150(2)$ \\
\hline $\mathrm{C} 5-\mathrm{C} 6$ & $1.383(2)$ & $\mathrm{C} 13-\mathrm{O} 13$ & $1.138(2)$ \\
\hline $\mathrm{C} 5-\mathrm{H} 5$ & $0.97(2)$ & $\mathrm{C} 14-\mathrm{O} 14$ & $1.133(2)$ \\
\hline $\mathrm{C} 12-\mathrm{Mn} 1-\mathrm{C} 11$ & $90.40(8)$ & $\mathrm{C} 4-\mathrm{C} 5-\mathrm{H} 5$ & $122.5(11)$ \\
\hline $\mathrm{C} 12-\mathrm{Mn} 1-\mathrm{C} 14$ & $89.72(8)$ & $\mathrm{C} 1-\mathrm{C} 6-\mathrm{C} 5$ & $123.10(14)$ \\
\hline $\mathrm{C} 11-\mathrm{Mn} 1-\mathrm{C} 14$ & $95.64(9)$ & $\mathrm{C} 1-\mathrm{C} 6-\mathrm{O} 2$ & $119.06(13)$ \\
\hline $\mathrm{C} 12-\mathrm{Mn} 1-\mathrm{C} 13$ & $90.80(8)$ & $\mathrm{C} 5-\mathrm{C} 6-\mathrm{O} 2$ & $117.74(14)$ \\
\hline $\mathrm{C} 11-\mathrm{Mn} 1-\mathrm{C} 13$ & $96.13(8)$ & $\mathrm{O} 1-\mathrm{C} 7-\mathrm{C} 2$ & $117.28(13)$ \\
\hline $\mathrm{C} 14-\mathrm{Mn} 1-\mathrm{C} 13$ & $168.21(8)$ & $\mathrm{O} 1-\mathrm{C} 7-\mathrm{C} 8$ & $119.36(15)$ \\
\hline $\mathrm{C} 12-\mathrm{Mn} 1-\mathrm{C} 1$ & $100.08(7)$ & $\mathrm{C} 2-\mathrm{C} 7-\mathrm{C} 8$ & $123.35(15)$ \\
\hline $\mathrm{C} 11-\mathrm{Mn} 1-\mathrm{C} 1$ & $169.49(7)$ & $\mathrm{C} 7-\mathrm{C} 8-\mathrm{H} 83$ & $110.0(14)$ \\
\hline $\mathrm{C} 14-\mathrm{Mn} 1-\mathrm{C} 1$ & $85.22(7)$ & $\mathrm{C} 7-\mathrm{C} 8-\mathrm{H} 82$ & $112.0(14)$ \\
\hline $\mathrm{C} 13-\mathrm{Mn} 1-\mathrm{C} 1$ & $83.09(6)$ & $\mathrm{H} 83-\mathrm{C} 8-\mathrm{H} 82$ & $106.7(19)$ \\
\hline $\mathrm{C} 12-\mathrm{Mn} 1-\mathrm{O} 1$ & $179.05(6)$ & $\mathrm{C} 7-\mathrm{C} 8-\mathrm{H} 81$ & $112.6(15)$ \\
\hline $\mathrm{C} 11-\mathrm{Mn} 1-\mathrm{O} 1$ & $90.46(7)$ & $\mathrm{H} 83-\mathrm{C} 8-\mathrm{H} 81$ & $110(2)$ \\
\hline $\mathrm{C} 14-\mathrm{Mn} 1-\mathrm{O} 1$ & $89.80(7)$ & $\mathrm{H} 82-\mathrm{C} 8-\mathrm{H} 81$ & $105(2)$ \\
\hline $\mathrm{C} 13-\mathrm{Mn} 1-\mathrm{O} 1$ & $89.51(6)$ & $\mathrm{O} 3-\mathrm{C} 9-\mathrm{O} 2$ & $122.64(15)$ \\
\hline $\mathrm{C} 1-\mathrm{Mn} 1-\mathrm{O} 1$ & $79.06(5)$ & $\mathrm{O} 3-\mathrm{C} 9-\mathrm{C} 10$ & $126.36(16)$ \\
\hline $\mathrm{C} 6-\mathrm{C} 1-\mathrm{C} 2$ & $115.00(13)$ & $\mathrm{O} 2-\mathrm{C} 9-\mathrm{C} 10$ & $111.00(15)$ \\
\hline $\mathrm{C} 6-\mathrm{C} 1-\mathrm{Mn} 1$ & $132.64(11)$ & $\mathrm{C} 9-\mathrm{C} 10-\mathrm{H} 102$ & $110.0(16)$ \\
\hline $\mathrm{C} 2-\mathrm{C} 1-\mathrm{Mn} 1$ & $112.36(11)$ & $\mathrm{C} 9-\mathrm{C} 10-\mathrm{H} 103$ & $111.9(17)$ \\
\hline $\mathrm{C} 3-\mathrm{C} 2-\mathrm{C} 1$ & $123.14(14)$ & $\mathrm{H} 102-\mathrm{C} 10-\mathrm{H} 103$ & $108(2)$ \\
\hline $\mathrm{C} 3-\mathrm{C} 2-\mathrm{C} 7$ & $122.76(14)$ & $\mathrm{C} 9-\mathrm{C} 10-\mathrm{H} 101$ & $108.0(16)$ \\
\hline $\mathrm{C} 1-\mathrm{C} 2-\mathrm{C} 7$ & $114.09(13)$ & $\mathrm{H} 102-\mathrm{C} 10-\mathrm{H} 101$ & $106(2)$ \\
\hline $\mathrm{C} 4-\mathrm{C} 3-\mathrm{C} 2$ & $118.92(15)$ & $\mathrm{H} 103-\mathrm{C} 10-\mathrm{H} 101$ & $113(2)$ \\
\hline $\mathrm{C} 4-\mathrm{C} 3-\mathrm{H} 3$ & $120.1(13)$ & $\mathrm{O} 11-\mathrm{C} 11-\mathrm{Mn} 1$ & $178.71(19)$ \\
\hline $\mathrm{C} 2-\mathrm{C} 3-\mathrm{H} 3$ & $120.9(13)$ & $\mathrm{O} 12-\mathrm{C} 12-\mathrm{Mn} 1$ & $177.51(16)$ \\
\hline $\mathrm{C} 3-\mathrm{C} 4-\mathrm{C} 5$ & $119.73(15)$ & $\mathrm{O} 13-\mathrm{C} 13-\mathrm{Mn} 1$ & $175.05(15)$ \\
\hline $\mathrm{C} 3-\mathrm{C} 4-\mathrm{H} 4$ & $122.1(12)$ & $\mathrm{O} 14-\mathrm{C} 14-\mathrm{Mn} 1$ & $176.86(19)$ \\
\hline $\mathrm{C} 5-\mathrm{C} 4-\mathrm{H} 4$ & $118.1(12)$ & $\mathrm{C} 7-\mathrm{O} 1-\mathrm{Mn} 1$ & $117.18(10)$ \\
\hline $\mathrm{C} 6-\mathrm{C} 5-\mathrm{C} 4$ & $120.10(15)$ & $\mathrm{C} 9-\mathrm{O} 2-\mathrm{C} 6$ & $117.20(12)$ \\
\hline $\mathrm{C} 6-\mathrm{C} 5-\mathrm{H} 5$ & $117.4(11)$ & & \\
\hline
\end{tabular}


Fig. 1

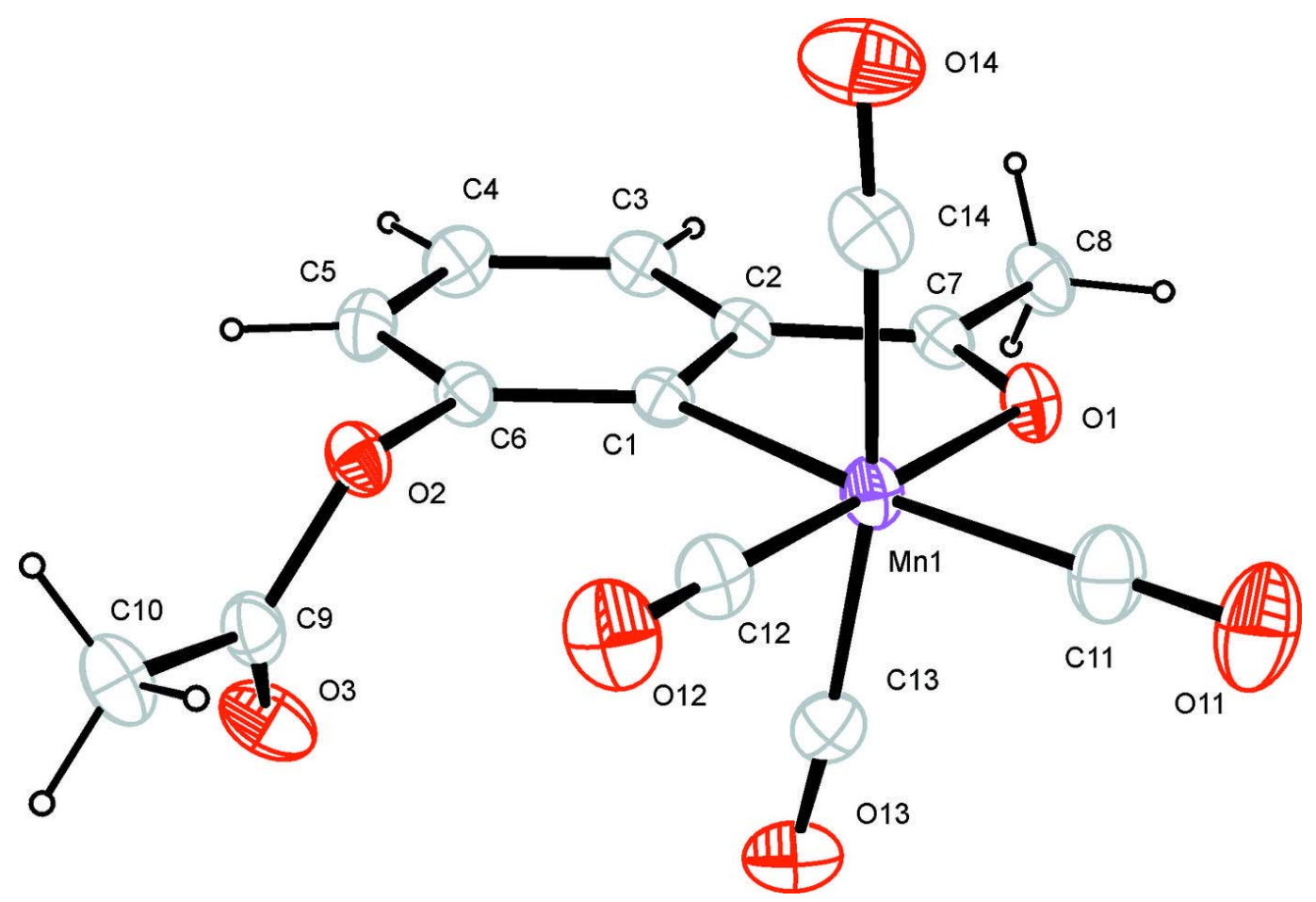

